\title{
AVANCES EN EL DESARROLLO DE UN SISTEMA DE MANUFACTURA ADITIVA BASADO EN STEP-NC
}

\author{
E. Rodriguez ${ }^{1 *}$; R. Bonnard ${ }^{2}$; A. Alvares ${ }^{1}$
}

1 Departamento de Ingeniería Mecánica y Mecatrónica, Universidad de Brasilia, Brasilia, Brasil

2 SENAI/SC Innovación para manufactura laser, sistemas de manufactura y sistemas embebidos, Florianópolis, Brasil

\author{
*efrainrg2009@gmail.com
}

Submitted at 15/12/2017 and accepted at 15/01/2018

\section{RESUMEN}

El nuevo estándar de control numérico, conocido como STEP-NC, es categorizado como el futuro de los sistemas avanzados de manufactura. Mayor flexibilidad e interoperabilidad son algunos de los potenciales beneficios que ofrece STEP-NC para enfrentar los desafíos del nuevo panorama industrial que se vislumbra con el advenimiento de la Industria 4.0. Mientras tanto, la programación orientada a objeto de STEP-NC ha sido parcialmente aplicada y desarrollada hasta el momento para los procesos de mecanizado (fresado, torneado...). Pero con los procesos de manufactura aditiva no ha sucedido lo mismo y el desarrollo es aún incipiente. Este trabajo presenta los avances en el desarrollo de un nuevo sistema de manufactura aditiva basado en STEP-NC, enfocándose particularmente sobre el desarrollo del modelo de información. EL modelo de actividades de aplicación en la nomenclatura del IDEF0 y el modelo de referencia de aplicación en EXPRESS son presentados. El concepto AM-layer-feature ha sido introducido para definir la feature de manufactura de los procesos aditivos basados en deposición de material capa por capa. Finalmente, se presenta un programa en STEP-NC generado desde el modelo EXPRESS, el cual puede ser implementado sobre un sistema de manufactura aditiva para validar el modelo propuesto.

\section{PROGRESSES IN THE DEVELOPMENT OF A STEP-NC COMPLIANT ADDITIVE MANUFACTURING SYSTEM}

\begin{abstract}
The new standard of numerical control, known as STEP$\mathrm{NC}$, is categorized as the future of the advanced manufacturing systems. Greater flexibility and interoperability are some potential benefits offered by STEP-NC to meet the challenges of the new industrial landscape that is envisaged with the advent of Industry 4.0. Meanwhile, STEP-NC object-oriented programming has been partially applied and developed for machining processes (milling, turning...). But with the processes of additive manufacturing has not happened the same and the development is still incipient. This work presents the advances in the development of a new STEP-NC
\end{abstract}

compliant additive manufacturing system, focusing particularly on the development of the information model. The application model activities in the IDEF0 nomenclature and application reference model in EXPRESS are presented. The AM-layer-feature concept has been introduced to define the manufacturing feature of additive processes based on material deposition layerby-layer. Finally, a STEP-NC program generated from the EXPRESS model is presented, which can be implemented on an additive manufacturing system to validate the proposed model.

KEYWORDS: additive manufacturing, STEP-NC, digital thread, interoperability, industry 4.0 


\section{INTRODUCCIÓN}

La nueva revolución industrial, denominada Industria 4.0, mantiene la promesa de aportar una mayor flexibilidad a los sistemas industrializados de manufactura, junto con la personalización masiva de productos y el aumento en la productividad de los procesos (Lasi et al., 2014). El principal objetivo es intervenir directamente en el proceso de desarrollo de los nuevos productos sobre el tríptico costo, tiempo y calidad. Así, este movimiento incentiva la automatización de la cadena de procesos a través de la digitalización y la producción en red, involucrando un conjunto de tecnologías de vanguardia que incluyen principalmente sistemas cibernéticos, datos masivos, internet de las cosas y computación en la nube, para lograr una completa transformación del sector industrial (Cardoso et al., 2017). El resultado de este nuevo panorama es una Fábrica Inteligente, donde los proveedores y clientes quedarán más cerca, ya que los pedidos de producción serán enviados por el cliente directamente a la máquina y los datos de producción se transferirán al socio de distribución en tiempo real. Esto hace que las empresas manufactureras sean más flexibles y eficientes en el desarrollo de productos altamente personalizados para satisfacer los requerimientos específicos del cliente.

En este nuevo panorama industrial, la Manufactura Aditiva (AM-Additive Manufacturing), también referida como Impresión 3D o Manufactura Rápida, es llamada a jugar un rol importante pues ofrece potenciales beneficios para el proceso de desarrollo de los nuevos productos, tales como reducción del tiempo de lanzamiento al mercado, alta personalización en los diseños para fabricación y reducciones en la cadena suministro (Huang et al., 2015). Los procesos de AM permiten producir objetos físicos directamente desde un modelo 3D virtual a través de la adición de material, en contraste con los procesos de manufactura substractiva. Su principal ventaja es la capacidad de producir partes con geometrías complejas sin la necesidad de múltiples setup y con el mínimo desperdicio de material. Desde sus inicios en los años 1980's, la AM fue destinada exclusivamente a la creación de modelos prototipo de productos. Sin embargo, gracias a los inmensos avances que ha experimentado en términos de velocidad, calidad, materiales y utilidades de máquina, ya es un hecho su extensión a la producción de partes funcionales directamente para un usuario final. Hoy en día, ha comenzado a ser empleada en un amplio rango de aplicaciones que incluyen la industria automotora y aeroespacial, la ingeniería biomédica, arquitectura, dispositivos electrónicos, comida, etc. (Negi, Dhiman e Sharma, 2016).

Con la incorporación de la AM en la producción real de partes funcionales, la demanda globalizada de productos fabricados con procesos aditivos es cada vez mayor y, así mismo, las exigencias en cuanto a la calidad del producto final son cada vez más altas. Fabricar un producto de calidad usando AM requiere de gran cantidad de información. Esta información incluye el modelo geométrico de la parte, información del material, información del proceso, datos de medición, etc. Por lo tanto, existe la necesidad de gerenciar apropiadamente la captura, almacenamiento y uso de toda esta información ( $\mathrm{Lu}$, Choi e Witherell, 2015). Esta labor ha sido dificultada principalmente por la inexistencia de un único formato estándar que contenga todas las informaciones que describen el ciclo de vida de un producto, lo cual es un problema que envuelve tanto la AM como los procesos de manufactura substractiva. Conforme esto, la integración de la cadena digital de los sistemas avanzados de manufactura, y específicamente de los sistemas de AM, a través de un único formato estándar, es el principal desafío a ser enfrentado si se piensa en incrementar la calidad de los productos y la productividad de los procesos (Bonnard, Mognol e Hascoët, 2010). Además, con el reciente advenimiento de la Industria 4.0, las tecnologías de la información y el concepto de Smart Factory, los sistemas de manufactura están siendo forzados a migrar hacia entornos más colaborativos e interoperables. Es decir, entornos de globalización donde la información de diseño y fabricación de una parte es manejada por diferentes trabajadores en distintas partes del mundo. Para lograr que los sistemas de manufactura puedan adaptarse a estos ambientes de globalización, es necesaria la integración de la cadena digital a través de un único formato estándar (Nassehi, A. Newman, S.T. Allen, 2006).

En un esfuerzo internacional por superar estos inconvenientes, surgen los estándares STEP (Standar for the Exchange of Product data models) y STEP-NC, amparados bajos las normas ISO 
10303 (Pratt, 2001) y ISO 14649 (“ISO 14649-1, 2003) respectivamente. El primero, proporciona un mecanismo de naturaleza neutral que define todas las informaciones de alto nivel relacionadas a un producto basado en features y permite el intercambio de estas informaciones entre los módulos de Diseño y Manufactura Asistida por Computador-CAD/CAM. El segundo, es una extensión del STEP para la definición de programas $\mathrm{NC}$, el cual representa un enlace para el intercambio de información del proceso entre el CAM y los sistemas de Control Numérico Computarizado-CNC. El STEP-NC es pensado para una nueva generación de controladores CNC inteligentes y con funciones avanzadas, lo cual es catalogado como el futuro de los sistemas avanzados de manufactura.

La integración de la cadena digital CAD/CAM/CNC basada en STEP-NC ya ha sido gradualmente desarrollada para procesos de mecanizado (Rauch et al., 2012; Suh et al., 2003; Toquica et al., 2017). En AM, este tema se encuentra aún en una fase prematura y debe ser desafiada con nuevos modelos de información y proposiciones de nuevas plataformas basadas en STEP-NC. En este contexto, el presente trabajo explora la aplicación de los estándares STEP y STEP-NC para soportar una cadena digital de alto nivel para sistemas de AM. Primero, se analiza la situación de la cadena digital actual de la AM. Enseguida, se resaltan las nuevas posibilidades con el estándar STEP-NC. Finalmente, el modelo de información es presentado y se describen los principales requerimientos para su implementación.

\section{MATERIALES Y MÉTODOS}

\subsection{La actual cadena digital de la AM}

Actualmente, para fabricar un producto a través de sistemas de AM, es necesario realizar conversiones entre diferentes formatos de archivo. La Figura 1 muestra una visión holística de la típica cadena digital usada actualmente en AM compuesta por cuatro fases principales: diseño; planeamiento de proceso; fabricación; y, post-procesamiento. En la primera fase, el modelo 3D de la parte es diseñado en un software CAD y exportado al software CAPP (Planeamiento de Proceso Asistido por Computador)/CAM usualmente en formato STL (Surface Tessellation Language) (Bonnard, Rodriguez e Alvares, 2018). Entonces, en el software CAPP/CAM (segunda fase) se selecciona la orientación óptima de fabricación y se proporcionan las estructuras de soporte a las superficies de la parte que están totalmente suspendidas en el aire (caso existan). Luego, se realiza el rebanado en capas con un espesor definido (slicing) del modelo 3D y se adicionan los parámetros de proceso tales como densidad del relleno, número de perímetros, volumen de deposición, etc. El software CAPP/CAM determina automáticamente los toolpath necesarios para cada una de las capas, y un post procesador genera instrucciones de máquina optimizadas (códigos G\&M) para un controlador CNC específico. En la tercera fase, la parte es manufacturada por la máquina de AM a través de la ejecución secuencial de las instrucciones generadas contenidas en el archivo CAM de la máquina específica. Finalmente, la parte fabricada puede ser inspeccionada para determinar errores dimensionales y/o geométricos, así como también puede ser aplicado un proceso de mecanizado para dar cavado superficial.

Se puede notar que la inexistencia de un único formato estándar para la transferencia de la información es el principal problema de esta cadena. Primeramente, el formato STL ha sido usado como estándar de facto para transferir la información del modelo CAD de la parte al sistema CAPP/CAM. Sin embargo, es sabido que el formato STL es tan solo una aproximación de la geometría exacta del modelo de la parte, representada por elementos triangulares discretos (Szilvśi-Nagy e Mátyási, 2003). Cada triangulo es definido por doce números de coma flotante correspondientes a su vector normal unitario y a sus tres vértices, donde el orden y la dirección están dados por la regla de la mano derecha. Esto ya representa problemas, pues pueden aparecer huecos en la geometría cuando un vértice es compartido por uno o más triángulos y pequeños errores de redondeo resultan en vértices que no coinciden exactamente. Aún peor, información de alto nivel como tolerancias, rugosidades, material, etc., necesaria para manufacturar un producto de calidad a través de un sistema de AM, no están presentes en este formato. Además, se ha evidenciado que el formato STL posee inconvenientes en la representación de la 
geometría tales como redundancia de información, escalabilidad deficiente, pérdida de elementos geométricos, unidades dimensionales no definidas, entre otros. Conforme esto, el formato STL se torna de lejos incompatible con el concepto de una cadena digital de alto nivel.

Por otro lado, el intercambio de información entre los sistemas CAPP/CAM y los sistemas de AM se hace a través de programas NC, los cuales continúan siendo definidos en el estándar ISO 6983 (ISO 6983-1, 1982), conocido como código G\&M. El lenguaje del ISO 6983 define comandos alfanuméricos con información limitada principalmente al movimiento de los ejes de la máquina CNC, despreocupándose por la información del proceso mismo. Además, cada fabricante es reluctante de definir instrucciones específicas para sus máquinas, lo cual representa un problema para la flexibilidad e interoperabilidad de los sistemas $\mathrm{CNC}$, debido a que por cada máquina se hace necesario un posprocesador diferente, lo que a su vez incurre en la necesidad de una persona especializada para operar cada máquina.

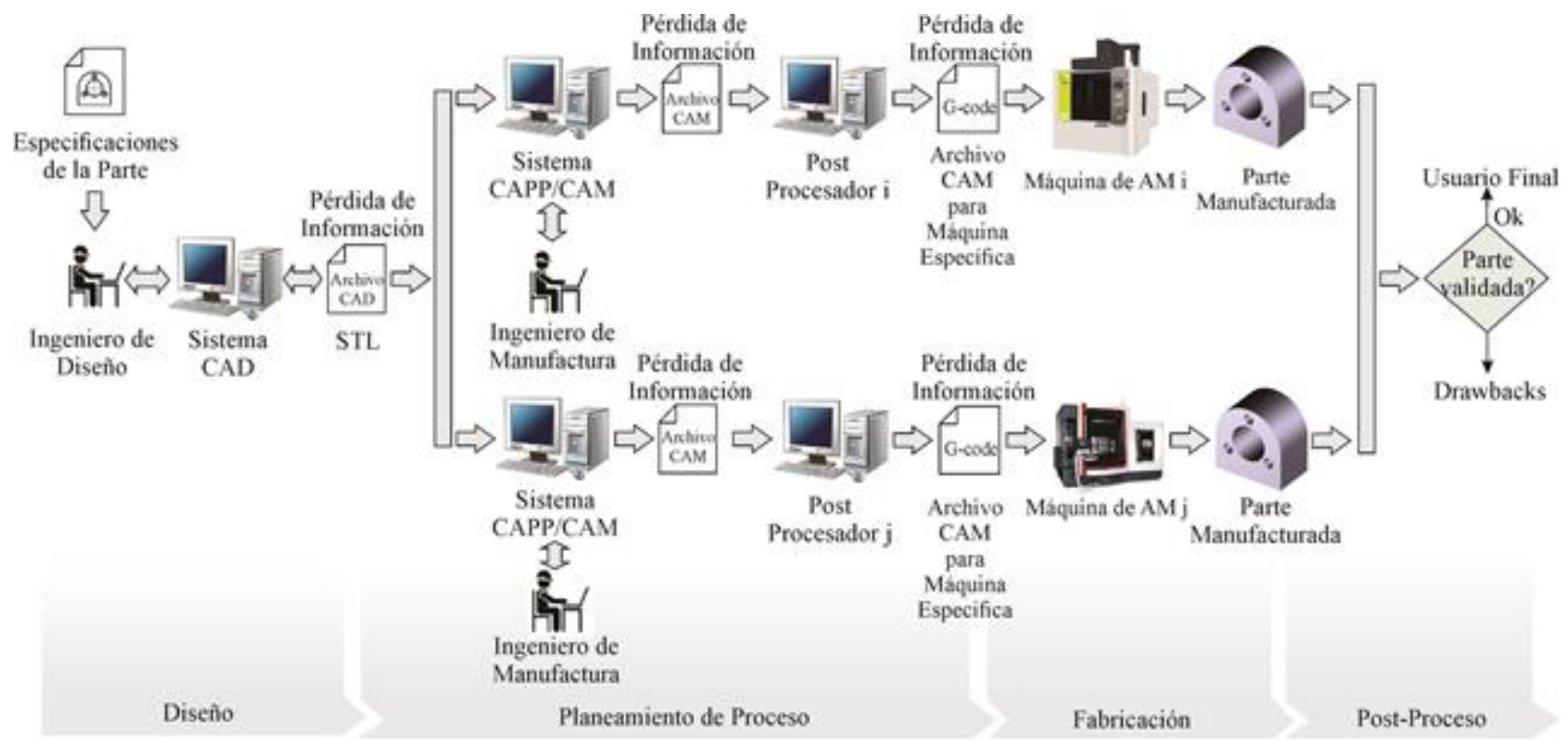

Figura 1. Situación actual de la cadena digital de los sistemas de AM.

Fuente: Elaboración propia.

Con base en el modelo de la Figura 1, se pueden resaltar los principales inconvenientes existentes en la cadena digital actual de la AM por la falta de un formato estándar:

- Pérdida de información.

- Flujo unidireccional de datos.

- Multiplicación de archivos - desestandarización.

- Inexistencia de información de alto nivel.

- Múltiples pos-procesadores.

- Inexistencia de comunicación entre los sistemas CNC.

- Falta de retroalimentación integrada con información de inspección de la parte fabricada.

- Dificultad para el almacenamiento y disposición de información en base de datos vía internet.

Hasta el momento, la cadena digital actual ha funcionado aceptablemente. Sin embargo, las demandas de la nueva industria, incluyendo el concepto de Industria 4.0, requieren de sistemas de manufactura con nuevas capacidades para adaptarse en ambientes de globalización. Esto es imposible de lograr con las soluciones actuales de formatos de archivos para intercambio de información del producto siendo fabricado. Por lo tanto, es necesario explorar nuevas posibilidades para soportar una cadena digital de alto nivel de AM a través de un único formato estándar. 


\subsection{El nuevo concepto de cadena digital para AM basada en STEP-NC}

Como fue expuesto antes, para superar los inconvenientes de la cadena digital actual de los sistemas de AM, los estándares actualmente usados deben ser reemplazados por un único estándar que contenga toda la información del ciclo de vida de un producto para ser manufacturado a través de procesos aditivos. En primera instancia y con el fin de superar los inconvenientes del formato STL, varios formatos han sido propuestos como posibles alternativas de solución. Algunos de los formatos considerados incluyen RPI (Rensselaer Polytechnic Institute) (Rock e Wozny, 1991), LMI (Layer Manufacturing Interface) (Kai, Jacob e Mei, 1997), CLI (Common Layer Interface) (Zeng et al., 2013), STH (Surface Triangles Hinted), CLF (Cubital Facet List) y recientemente, el formato AMF (Additive Manufacturing Format) (Nassar e Reutzel, 2013) y el formato 3MF (3D Manufacturing Format), pero estos formatos no son totalmente adecuados a todos los requerimientos de los procesos industriales de $\mathrm{AM}$, y mucho menos permiten la transferencia de datos de manufactura hasta el nivel del controlador CNC.

La falta de un único formato estándar es un problema que persiste tanto sobre la cadena digital de la AM como de los procesos de mecanizado, lo cual ha sido por mucho tiempo uno de los mayores inconveniente enfrentado por la industria manufacturera (Brunnermeier e Martin, 1999). En vista de esto, desde mediados de los años 1980's se ha iniciado un esfuerzo unificado que envuelve académicos e industriales de la comunidad internacional con el fin de desarrollar un poderoso conjunto de estándares, todos bajo la norma ISO 10303 (Pratt, 2001), también conocida como STEP. El objetivo de STEP es proporcionar un lenguaje común para describir todos los aspectos técnicos de un producto a lo largo de su ciclo de vida. La naturaleza neutra del este estándar lo tornan adecuado para la transferencia de datos entre programas de software CAD y CAM, independientemente del sistema en particular. Ahora, el nuevo el nuevo estándar de control numérico basado en STEP, conocido como STEP-NC, es una extensión de los modelos del ISO 10303 para incluir información del proceso de manufactura y de la máquina-herramienta. STEP-NC es pensado para facilitar el intercambio de datos entre sistemas CAPP/CAM y sistemas CNC, y proporcionar un modelo de datos orientado a objeto para la siguiente generación de controladores inteligentes con funciones avanzadas. Hoy en día, existen dos versiones de STEP-NC bajo desarrollo dentro de la ISO: la primera es ISO 14649 (ISO 14649-1, 2003) - modelo ARM (Application Reference Model) siendo desarrollada por el comité ISO TC184/SC1 y la segunda es ISO 10303-238 (ISO 10303-238, 2007) - AIM (Application Interpreted Model) siendo desarrollada por el comité ISO TC184/SC4. ISO 10303-238 coloca todo lo que hay en el ARM dentro del AIM para lograr una mejor integración con otros recursos de STEP (Xu et al., 2005). Kramer et al. (Kramer et al., 2006) demostraron que ambas versiones de STEP-NC pueden ser implementadas. Así, los esfuerzos para el desarrollo de modelos de información y plataformas basadas en STEP-NC se han enfocados principalmente sobre los procesos de mecanizado (Cha et al., 2016; Suh et al., 2003; Xú, Anwer e Lavernhe, 2014; Zhang et al., 2011), inclusive con el uso de internet como medio de comunicación (Álvares et al., 2016).

STEP-NC es ahora categorizado como el gran desafiador de las deficiencias de la cadena digital de la AM. Nuevas posibilidades para soportar una cadena digital de alto nivel totalmente estandarizada son dadas por este nuevo estándar. La Figura 2 ilustra el concepto de una cadena digital de AM basada en STEP-NC integrada y estandarizada, la cual es soportada sobre un único formato de archivo para el intercambio de información desde el diseño hasta el controlador CNC. Así, el único archivo STEP-NC de AM es enriquecido con información desde cada unos de los sistemas CAx. Grandes beneficios pueden ser logrados a partir de la nueva cadena digital listados a continuación:

- Se elimina la multiplicidad de archivos y la pérdida de información.

- La información de alto nivel y la información del proceso pueden ser conectadas con la información del modelo 3D de la parte en un único archivo.

- Se habilita el flujo bidireccional de información los sistemas CAx. 
- No hay necesidad de pos-procesadores, por lo tanto un solo ingeniero de manufactura puede operar varias máquinas con diferentes recursos a la vez.

- Existe interoperabilidad entre los sistemas CNC de AM.

- Se habilita la retroalimentación del sistema con un módulo inspección en malla cerrada (Riaño et al., 2017).

- Se facilita la disposición de información sobre una base de datos en la nube.

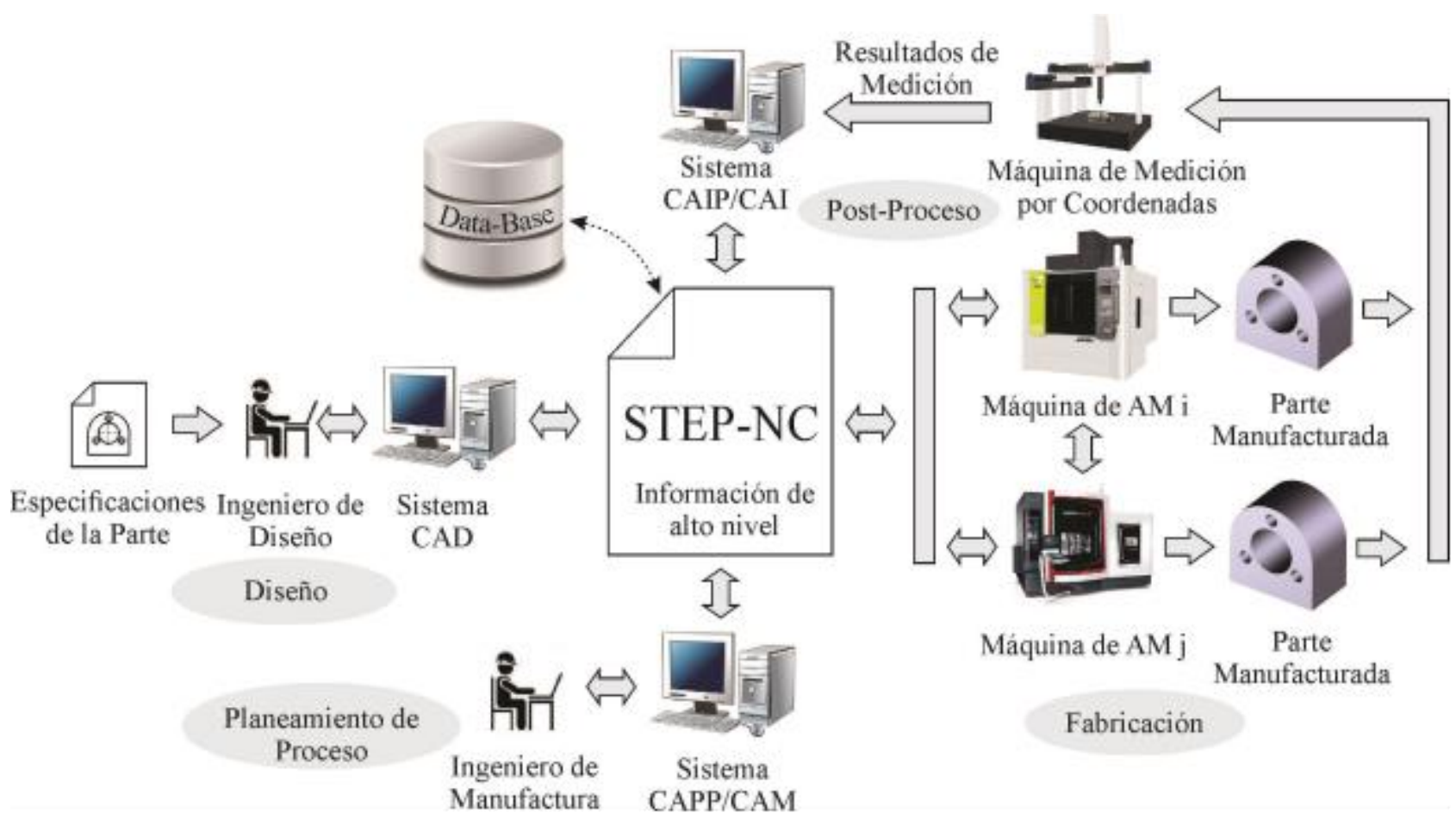

Figura 2. Nuevo concepto para la cadena digital de la AM basada en STEP-NC.

Fuente: Elaboración propia.

\subsection{El por qué de esta propuesta}

Con el incremento en la complejidad de la parte y de los procesos digitales, la integración de información geométrica con las informaciones adicionales de especificación (material, tolerancias, rugosidades, etc.) está siendo cada vez más y más importante a la hora de mejorar la calidad de los productos y la eficiencia de los procesos. En este punto, se hace notoria la necesidad de un único y robusto formato estándar que contenga toda la información de alto nivel de un producto descrita a lo largo de su ciclo de vida. Carleberg (Carleberg, 1994) es el primero en proponer el formato STEP para conectar la información del proceso con la información del modelo de la parte en procesos de AM. Pratt (Pratt, 1995) sugiere adaptar el formato STEP para soportar información del diseño de partes a ser fabricadas con AM. Kumar y Dutta (Kumar e Dutta, 1997) evalúan los requerimientos y recursos necesarios para la trasferencia de información entre sistemas CAD y sistemas de AM, y concluyen que el STEP es una buena solución. Otros autores (Danjou e Koehler, 2008; Jee e Lee, 1999) también han afirmado que el STEP es el mejor formato para AM, ya que permite asociar el modelo geométrico de la parte con información de especificaciones e información del mismo proceso.

Ryou et al. (Ryou et al., 2006) proponen un completo modelo de información en EXPRESS para representar el modelamiento de partes para ser fabricadas con procesos de AM. La metodología de tal propuesta consiste en dividir la información de diseño de la parte en cuatro categorías:

- sub_part_geo_design-contiene la información de las features geométricas del modelo CAD de la parte. 
- sub_part_non_geo_design- contiene la información sobre las features no-geométricas de la parte (tolerancias, rugosidad, material, color).

- sub_part_process_history-contiene un historial de los trabajos previos.

- sub_part_locally_controlled- esta categoría es opcional y hace referencia a la información de partes multi-colores.

La propuesta de Ryou et al. pretende incluir la AM dentro de la norma ISO 14649 e plantean con este fin, el desarrollo de un pre-procesador para la implementación del modelo de información.

En Francia, el equipo del Profesor Hascoët (Bonnard, Mognol e Hascoët, 2010) ha concernido sobre la necesidad de un enfoque global con una cadena digital AM que envuelva la representación de datos de fabricación hasta el nivel del controlador NC. Por lo tanto proponen una nueva cadena digital descrita por un modelo de información jerarquizado basado en STEP-NC. Dicha cadena permite un intercambio bidireccional de datos de alto nivel de AM entre sistemas CAD-CAM-CNC y ofrece potenciales oportunidades para el desarrollo de nuevos sistemas de AM avanzados y flexibles (Bonnard, Mognol e Hascoët, 2009, 2010). La propuesta del equipo del Profesor Hascoët se hizo de acuerdo con la intención del comité ISO TC 184 / SC 1 de incluir los procesos AM dentro de la norma ISO 14649. En realidad, los procesos aditivos han sido propuesta como la Parte 17 de ISO 14649 (ISO/AWI14649-17, 2016), el cual es un proyecto aún en desarrollo. Esta etapa crea una oportunidad para la proposición de nuevos modelos de datos de AM con STEP-NC y para el desarrollo de una plataforma STEP-NC de AM enfocada a la implementación para validar los modelos propuestos.

Las ventajas de la nueva cadena digital permitirían una completa adaptación de la AM a las exigencias de la nueva industria. Sin embargo, así como son grandes los beneficios aportados por STEP$\mathrm{NC}$ a la cadena digital de los procesos, también se hace mayor la complejidad para su implementación en los actuales sistemas. Por lo tanto, para enfrentar tal complejidad y lograr una cadena digital de AM completamente soportada sobre STEP-NC, los cambios sobre ésta deben ser realizados gradualmente. Rauch et al.(Rauch et al., 2012) proponen un método basado en tres niveles de implementación para adaptar gradualmente los sistemas de manufactura al nuevo estándar STEP-NC: en el primer el control de sistema es indirecto, es decir, el programa en STEP-NC es convertido a código G para que pueda ser entendido por los controladores ya existentes; en el segundo nivel ya existe un control directo, donde los tool-path y las operaciones son ejecutadas por la máquina directamente desde el programa STEP-NC; en el tercer nivel el controlador el sistema tiene la capacidad de realizar tareas de manera inteligente y autónoma, el cual sería el nivel a alcanzar por todos los sistemas avanzados de manufactura. En AM el desarrollo e implementación de modelos de información basados en STEP-NC es aún incipiente. Esta situación crea la oportunidad para la proposición de nuevos modelos y plataformas para AM con STEP$\mathrm{NC}$, que es por lo que este trabajo pretende contribuir con la concepción de un modelo de datos que pueda ser implementado sobre una plataforma de AM. El modelo es descrito en la siguiente sección.

\section{RESULTADOS}

\subsection{Modelo de actividades de aplicación para describir el sistema}

Un Modelo de Actividades de Aplicación (AAM-Application Activities Model) permite informar sobre el flujo de información entre las distintas actividades de un proceso. El modelo AAM es formalizado en la norma ISO 14649-1 (ISO 14649-1, 2003). Este modelo utiliza la nomenclatura IDEF0 para la representación de actividades funcionales. Cada actividad transforma una data de entrada en una data de salida a través de mecanismos y controles. Esta representación resulta en diagramas multicapas donde una actividad puede contener sub-actividades, y a su vez éstas contener otras sub-actividades.

La Figura 3 muestra el contexto para una cadena digital de AM con STEP-NC. Este primer diagrama representa la actividad A0 según la nomenclatura IDEF0, la cual es descompuesta en tres actividades principales que representan los módulos CAD, CAPP/CAM y CNC. Cada módulo es explicado en detalle. 


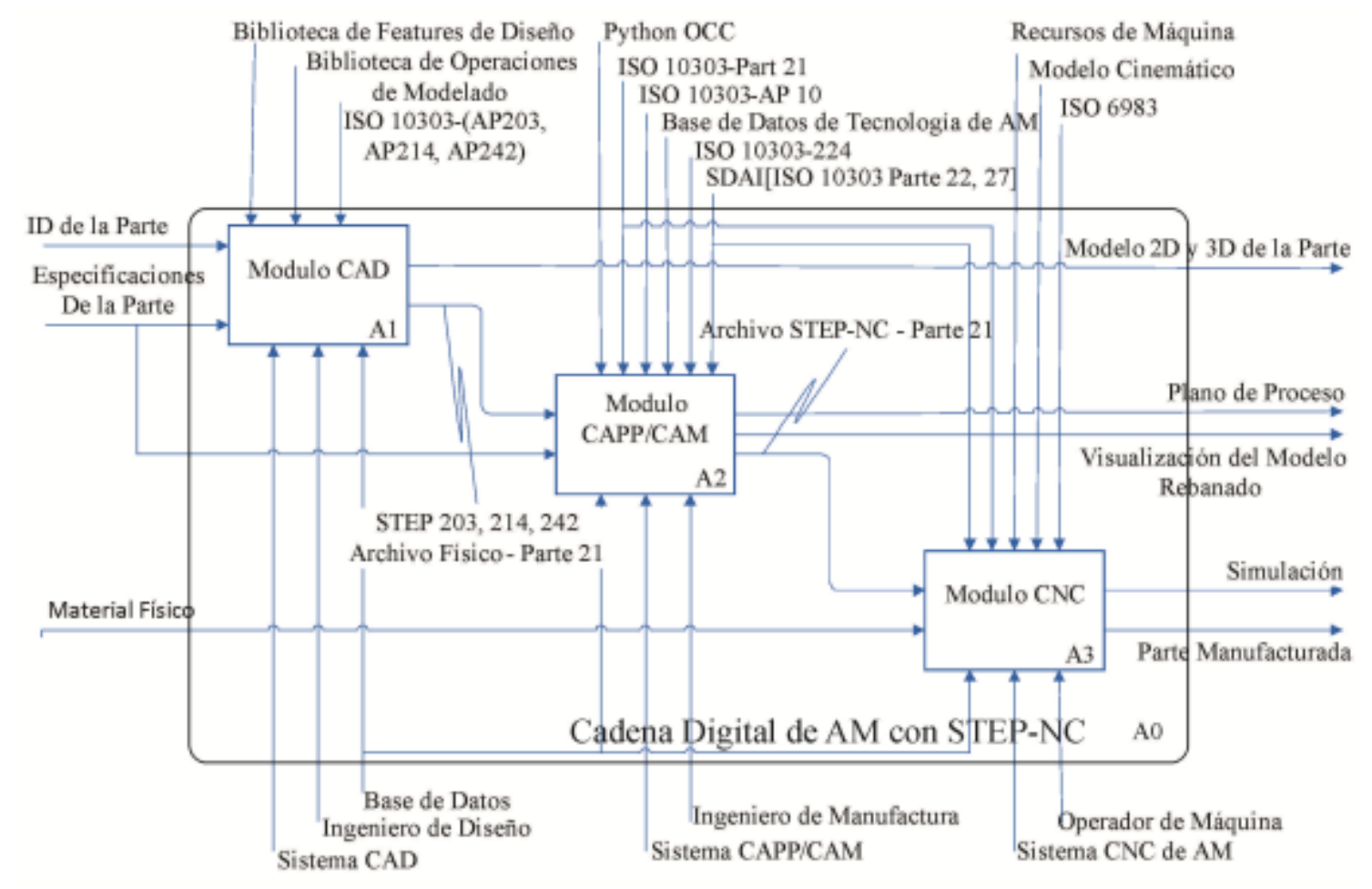

Figura 3. Actividad A0: Contexto para una cadena digital de AM con STEP-NC. Fuente: Elaboración propia.

En el módulo CAD (Actividad A1) ocurre la tarea de diseño y modelado 3D de la parte que se pretende fabricar con AM. Esta tarea es realizada a través de un sistema de software CAD. Este software puede ser cualquier sistema comercial disponible en el mercado, ya sean licenciados o libres, siempre y cuando tengan soporte para recursos de ISO 10303 (por ejemplo Solidworks, Catia, etc.). En el dominio CAD, los Protocolos de Aplicación (APs) de STEP más comunes son: AP203-configuration controlled $3 D$ designs of mechanical parts and assemblies (ISO 10303-203:2011, 2011); AP214-core data for automotive mechanical design processes (ISO 10303-214:2010, 2010); y, AP242 managed model-based $3 D$ engineering (ISO 10303-242:2014, 2014). El archivo STEP con la información de diseño de la parte es la data de entrada al sistema CAPP/CAM.

El planeamiento del proceso y la preparación para la producción son tareas esenciales previas a la fabricación de una parte con AM. Estas tareas son llevadas a cabo en el módulo CAPP/CAM (Actividad A2). En la Figura 4 se presenta una descomposición del módulo CAPP/CAM en cuatro subactividades concernientes al planeamiento del proceso y preparación para la fabricación de una parte por AM. Este módulo recibe el archivo STEP con la información geométrica y topológica de la parte. En el formato STEP la data de la parte es representada por features. Las features incluyen datos de representación de contorno como shells, caras, bucles de borde, vértices; datos geométricos de superficie tales como planos, cilindros, conos, toroides, esferas y curva geometría tales como líneas, círculos, $B$-splines, elipses. Esta información es reconocida en la actividad A21 a través de herramientas Python OCC (Paviot, 2014). Desde esta actividad salen las features geométricas que en la siguiente actividad (A22) son rebanadas (slicing) en capas con un determinado espesor (features de AM concepto explicado en la siguiente sub-sección). El conjunto de capas pasa a la siguiente actividad (A23) en formato SVG (Consortium World Wide Web, 1999) para generar el plano de proceso (workplan). Dentro de esta actividad se crean los workingsteps de AM, donde cada AM-workingstep tiene asociadas una $A M$-feature y una operación. Además se selecciona el tipo de tecnología de AM usada, recursos de máquina y parámetros de proceso a partir de las especificaciones de diseño de la parte (tales como tolerancias, rugosidades, material, etc.) a través de una base de conocimiento 
especializada. Los toolpath también son generados para cada una de las capas (AM-features). En seguida, en la actividad (A24) se genera el programa STEP-NC con la secuencia de los $A M$ workingsteps referenciando ISO 10303-Parte 21 (ISO 10303-21:2016, 2016) y JSDAI (ISO 10303-Parte 22, 27) (ISO/TS 10303-27:2000, 2000; ISO 10303-22:1998, 1998). La salida de esta actividad es el archivo físico STEP-NC en Parte 21 con el programa para fabricar una pieza con un proceso de AM.

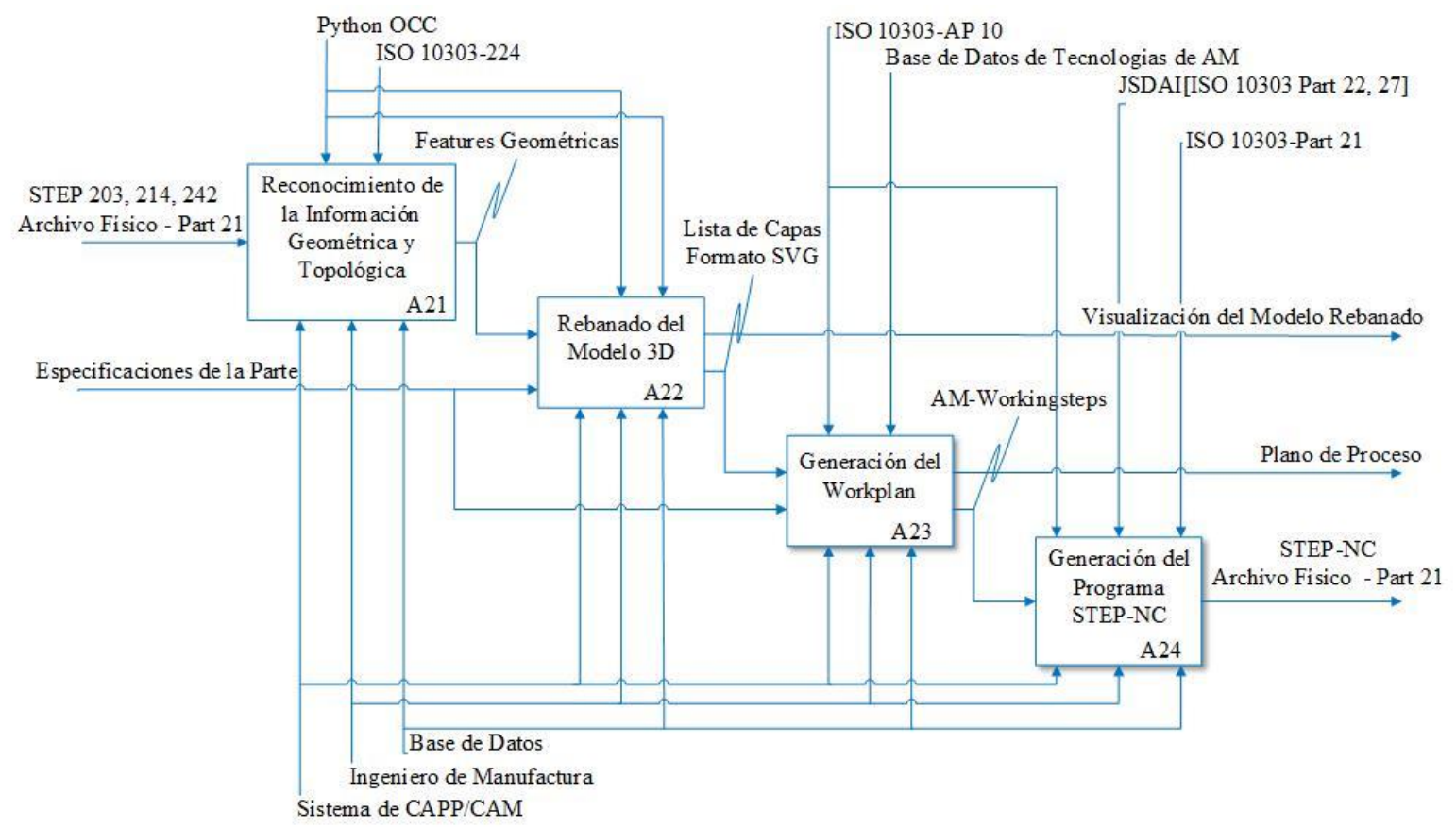

Figura 4. Actividad A2: Descomposición del módulo CAPP/CAM Fuente: Elaboración propia.

El archivo STEP-NC pasa entonces al módulo CNC (Actividad A3), en el cual se tiene un sistema CNC de AM con control indirecto, de acuerdo con el primer nivel del método de Rauch et al.(Rauch et al., 2012), donde las informaciones asociadas AM-workingstep son convertidas a código G a través de una aplicación de software adaptador. El código $\mathrm{G}$ generado es fácilmente entendido por los controladores existentes en las máquina de AM actuales. La interpretación de los comandos de código G es realizada a través de un firmware embebido en el controlador, el cual genera las señales sobre los drivers de los motores para generar el movimiento de los ejes de la máquina. Esto permite realizar una rápida implementación del modelo generado para el planeamiento del proceso en el módulo CAPP/CAM. Al final es posible obtener una parte manufacturada con un proceso de AM, cuya etapa de planeamiento ha sido llevada a cabo con base en un modelo STEP-NC.

Hasta el momento se ha descrito la arquitectura funcional del sistema global. Sin embargo, para poder pasar a la implementación es necesario construir un Modelo de Referencia de Aplicación (ARMApplication Reference Model) en lenguaje EXPRESS, pero antes se introduce un concepto para feature de AM.

\subsection{Definición de un concepto de feature para AM}

El estándar STEP-NC usa el concepto de programación basada en funciones para representar un plano de proceso que incorpora información sobre las features de fabricación, la descripción de la máquina-herramienta y la tecnología y las operaciones a ejecutar. Dentro del modelo ARM de STEP$\mathrm{NC}$, la pieza que se está fabricando (workpiece) se describe mediante features de fabricación que se definen según el tipo de proceso. Por lo tanto, para los procesos de mecanizado se han definido las features mecanizado (tales como holes, pockets, slot...) basadas en las features de fabricación de ISO 
10303-AP224 (Mechanical product definition for process planning using machining features) (ISO 10303-224:2006, 2006). Pero las features de mecanizado no están adaptadas para procesos aditivos y, de hecho, todavía no se ha establecido formalmente un concepto de feature para AM. Por lo que definir el concepto de feature de AM es un paso esencial hacia el desarrollo de un nuevo modelo de datos. Desde el entendimiento de los autores, la feature de AM corresponde a cada capa del modelo 3D rebanado de la parte que se está fabricando. Es decir, una feature de AM es referenciada como una capa, donde cada capa se define geométricamente mediante contornos externos e internos además del espesor. Este concepto de AM-layer-feature se ilustra en la Figura 5. Luego, la nueva feature-AM-layer debe ser modelada dentro del ARM para AM.
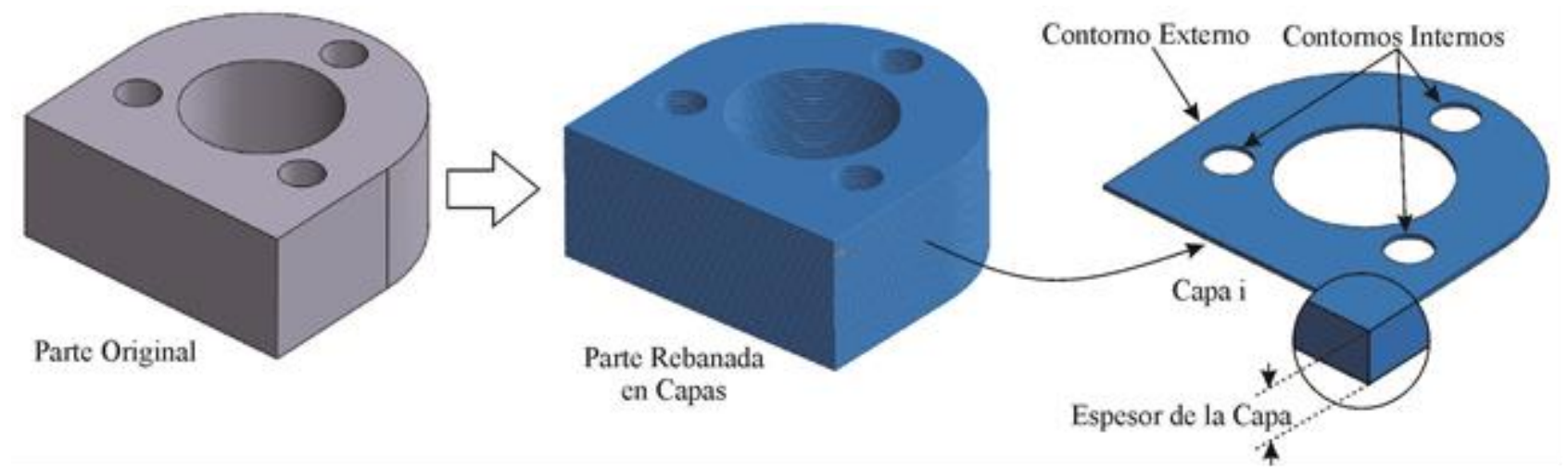

Figura 5. Ilustración del concepto de AM-layer-feature.

Fuente: Elaboración propia.

\subsection{Modelo de referencia de aplicación}

El ARM proporciona un modelo de datos orientado a objeto que usa el concepto de programación basada en feature para representar la información a ser usada por el controlador CNC. Tal información incluye features de manufactura, tecnología y herramientas a ser usadas, secuencia de operaciones y plano de proceso, la cual es especificada a través de un método de descripción que facilita la implementación. El lenguaje EXPRESS es un método de descripción de datos formalizado como Parte 11 de STEP (ISO 10303-11:2004, 2004), el cual permite especificar toda la información técnica de un producto de manera consistente, precisa y compacta, usando un estilo de modelo de datos basado en la relación Entidad-Atributo. De esta manera en EXPRESS un objeto es llamado Entidad y las propiedades correspondientes a dicho objeto son llamadas atributos. La relación entre entidades es dada por el grado de parentesco que existe entre ellas; así, una entidad puede ser supertipo de una entidad inferior y/o ser subtipo de una superior. EXPRESS legible y entendible tanto para humanos como para computadores, y su manera de representación de datos puede ser textual con una semántica léxica o gráfica con diagramas ilustrativos (esta última llamada EXPRESS-G, también dada en Parte 11).

En la Figura 6, se presenta parte del modelo de referencia de aplicación para un proceso de AM, el cual ha sido programado en lenguaje EXPRESS y luego convertido a EXPRESS-G para mayor compresión de la jerarquía de las entidades. La estructura principal del modelo es referencia desde el esquema del modelo dado en ISO 14649-10 (ISO 14649-10, 2004). Este modelo contiene elementos de datos que definen una interfaz entre el software de programación y el controlador de la máquina. A un proyecto de AM son asociados un Workplan principal y una lista de ejecutables. Además, también se incluye una AM-Workpiece con la descripción de la geometría de la parte. Un Workplan contiene una lista una lista con la secuencia de AM-Workingsteps, donde cada AM-Workingstep tiene asociada una operación a ser aplicada sobre una AM-feature. A su vez, para cada operación de define el tipo de tecnología y herramienta usada, las funciones de máquina y la estrategia de adición de material. 
El concepto de AM-layer-feature ha sido modelado e introducido dentro del ARM propuesto en este enfoque para procesos de AM. Las entidades en EXPRESS mostradas en la Figura 6 corresponden a la descripción a la AM-layer-feature. Así mismo, en el modelo en EXPRESS-G de la misma figura se resalta en rojo el concepto de AM-layer-feature como subtipo de la entidad manufacturing-feature. La entidad AMlayer-feature tiene asociado un plano de construcción de la capa con su respectivo espesor y la dirección de construcción. De igual forma, la AM-layer-feature contiene una lista de contornos internos y externos que representan los bordes de material solido dentro de una capa. Cada contorno debe ser cerrado y no debe intersecarse consigo mismo o con algún otro contorno. El tipo de contorno (interno o externo) es dado por su dirección, la cual es definida a través del atributo contour_direction. Así, los contornos internos son definidos en la dirección a favor de las manecillas del reloj y los contornos externos en contra de la dirección de las manecillas del reloj. Cada contorno puede ser también llamado polígono, el cual es definido por una polilínea a través de un conjunto de puntos cartesianos. En este enfoque, se ha escogido la entidad de polilínea (polyline) dada en ISO 10303 Parte 42 (ISO 10303-42:2014, 2014) como un tipo de curva para la representación de los contornos de una capa (AM-layer-feature).
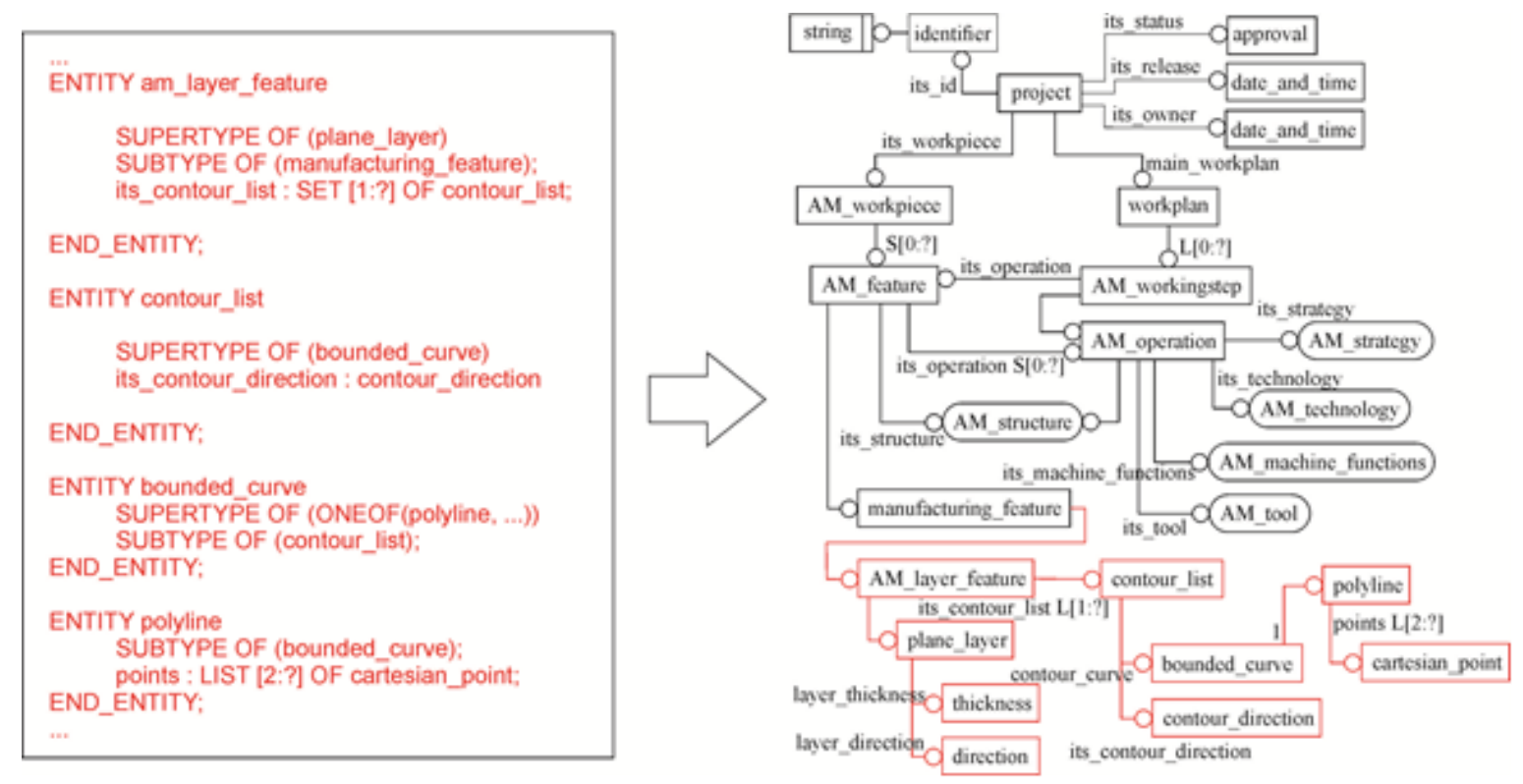

Figura 6. Fragmento del ARM de AM en EXPRESS y EXPRESS-G.

Fuente: Elaboración propia.

Teniendo el modelo lógico de entidades en EXPRESS, es necesario un método de implementación para instanciar las entidades del modelo de datos en un archivo físico que pueda ser leído por sistema de manufactura. El clear text encoding of the exchange structure como es definido en la Parte 21 de STEP, o simplemente llamado archivo físico Parte 21, es típicamente usado para registrar y almacenar instancias de entidades en archivos físicos de computador. Un archivo en Parte 21 es seccionado en un encabezado $(H E A D E R)$ y un cuerpo de datos (DATA). La sección de encabezado contiene información de identificación general a cerca del archivo, persona, organización y fecha. La sección de DATA incluye todas las entidades instanciadas desde el modelo de datos correspondientes al programa a ser ejecutado para la fabricación de una parte. Un ejemplo de un fragmento de programa en STEP-NC generado desde el ARM para fabricar una parte en material ABS usando un proceso aditivo basado en deposición de material fundido, es presentado en la Figura 7.

El archivo físico en Parte 21 de un programa en STEP-NC puede entonces ya ser implementado sobre un sistema de AM. Actualmente, el LaDPRER (Laboratório de Desenvolvimento de Produto: Protipagem Rápida e Engenharia Reversa) de la Universidad de Brasilia, se está trabajando en el desarrollo de una plataforma abierta de AM basada en un robot de cinemática paralela delta linear (Rodriguez, Riano Jaimes e Alvares, 2017), la cual funcionará como plataforma de implementación de los modelos STEP-NC. En primera instancia esta plataforma usará un método de control indirecto. Es decir, utilizando una 
aplicación de software adaptador que convierte un programa en STEP-NC a instrucciones de código G. Se hace uso de una tarjeta controladora basada en tecnología de desarrollo abierto de Arduino, a la cual se le carga un firmware que fácilmente interpreta los comandos de código $\mathrm{G}$ y genera las respectivas acciones sobre los actuadores de la máquina. Al final, la combinación de los movimientos de los actuadores de la máquina producto de la resolución de la cinemática inversa, permiten el posicionamiento del efector final (hotend) del robot para la deposición del material plástico fundido sobre la base de impresión.

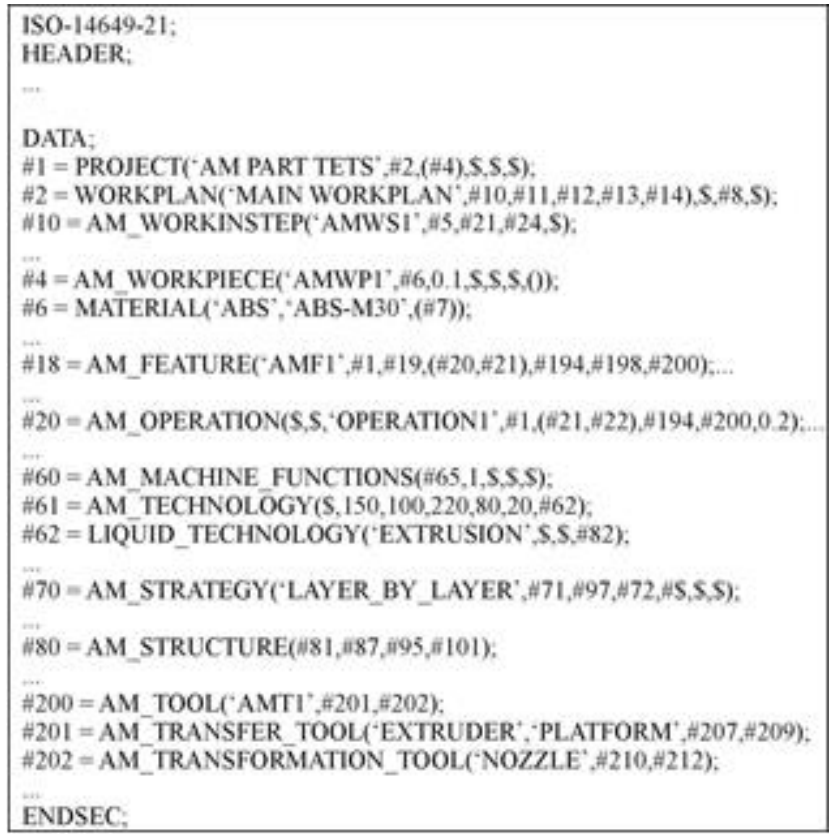

Figura 7. Ejemplo de un programa de AM en STEP-NC Parte 21 Fuente: Elaboración propia.

\section{CONCLUSIONES}

Este trabajo ha presentados los avances en el desarrollo de un nuevo sistema de AM basado sobre estándar STEP-NC, enfocándose particularmente sobre la propuesta de un modelo de información que pueda ser implementado sobre un sistema de AM por deposición de material fundido. La proposición se da dentro del contexto del nuevo panorama industrial-Industria 4.0, donde la interoperabilidad de los sistemas de manufactura computarizados y la transparencia de la información generada, intercambiada y usada para fabricar un producto juegan un rol preponderante. En este contexto, STEP-NC se convierte en el gran desafiador de las exigencias impuestas por la Industria 4.0 a los sistemas avanzados de manufactura y promete ser la solución a los problemas persistentes en hasta ahora en intercambio de datos de un producto a lo largo de su ciclo de vida.

En este trabajo, el estado actual de la cadena digital AM ha sido minuciosamente revisado a fin de listar los principales inconvenientes. El formato STL y el estándar de código $G$ se tornan incompatibles con la idea de una cadena digital de alto nivel para procesos de AM. Se ha demostrado que una completa cadena digital de alto nivel integrada y estandarizada es posible con el uso de STEPNC, y se han resaltado los beneficios potenciales de dicha cadena. Sin embargo, para lograr que los sistemas de manufactura aditiva puedan empezar a trabajar con el nuevo estándar, los cambios deben realizarse gradualmente y el desarrollo de proyectos piloto para demostrar los beneficios de la programación con STEP-NC debe ser promovido.

Con el modelo propuesto, los datos de diseño de la pieza se transfieren del software CAD al software CAPP/CAM utilizando un archivo STEP y eliminando la necesidad del formato STL. El modelo 3D de la pieza se rebana en capas directamente desde el archivo STEP utilizando las 
herramientas de PythonOCC. En este abordaje, el concepto de AM-layer-feature se introduce para definir las features de manufactura de procesos aditivos basados en la adición de capas planas sucesivas. El principal aporte de esta propuesta ha sido representar el concepto de feature de AM abordado usando la entidad de polilínea. También, se mostró un ejemplo de programa en STEP-NC para manufacturar una parte a través de un proceso de AM por deposición de material generado desde el ARM propuesto.

Como trabajos a futuros, se pretende el desarrollo de un controlador con capacidad para interpretar directamente un programa en STEP-NC y ejecutar las respectivas operaciones de fabricación. Así mismo, se pretende extender este enfoque sobre plataformas de manufactura hibrida combinando procesos aditivos y substractivos para manufactura complementada. También se tienen previsto usar los modelos de información de STEP-NC para disponibilizar informaciones de proceso en bases de datos en la nube a través del protocolo MTconnect.

\section{REFERENCIAS}

ÁLVARES, A. J. et al. A web-based STEP-NC-compliant architecture for low cost 3D part manufacturing. International Journal of Manufacturing Research, v. 11, n. 1, p. 1, 2016.

BONNARD, R.; MOGNOL, P.; HASCOËT, J. Y. Integration of Rapid Manufacturing processes in a high-level numerical chain. 4th International Conference on Advanced Research in Virtual and Rapid Prototyping, p. 595-601, 2009.

BONNARD, R.; MOGNOL, P.; HASCOËT, J.-Y. A new digital chain for additive manufacturing processes. Virtual and Physical Prototyping, v. 5, n. 2, p. 75-88, jun. 2010.

BONNARD, R.; RODRIGUEZ, E.; ALVARES, A. J. An Advanced STEP-NC Platform for Additive Manufacturing. In: Industrializing Additive Manufacturing - Proceedings of Additive Manufacturing in Products and Applications AMPA2017. Cham: Springer International Publishing, 2018. p. 127-136.

BRUNNERMEIER, S. B.; MARTIN, S. A. Interoperability cost analysis of the US automotive supply chain: Final report. Research Triangle Institute, n. 7007, 1999.

CARDOSO, W. et al. Digital manufacturing, industry 4.0, clould computing and thing internet: Brazilian contextualization and reality. Independent Journal of Management \& Production, v. 8, n. 2, p. 459, 1 jun. 2017.

CARLEBERG, P. Product Model Driven Direct Manufacturing. The SFF Symposium, University of Texas, p. 270-276, 1994.

CHA, J.-M. et al. A roadmap for implementing new manufacturing technology based on STEP-NC. Journal of Intelligent Manufacturing, v. 27, n. 5, p. 959-973, 4 out. 2016.

CONSORTIUM WORLD WIDE WEB. Scalable Vector Graphics - SVG Format. 1999.

DANJOU, S.; KOEHLER, P. Bridging the Gap between CAD and Rapid Technologies Exigency of Standardized Data Exchange. 12th European Forum on Rapid Prototyping, 2008.

HUANG, Y. et al. Additive manufacturing: current state, future potential, gaps and needs, and recommendations. Journal of Manufacturing Science and Engineering, v. 137, n. 1, p. 14001, 2015.

ISO 14649-1: Industrial automation systems and integration - Physical device control — Data model for computerized numerical controllers —Part 1: Overview and fundamental principles. 2003.

ISO 14649-1:2003. Industrial automation systems and integration - Physical device control - Data model for computerized numerical controllers - Part 1: Overview and fundamental principles. [s.d.].

ISO 14649-10:2004. Industrial automation systems and integration - Physical device control - Data model for computerized numerical controllers-Part 10: General process data. [s.d.].

ISO 6983-1:1982. Numerical control of machines - Program format and definition of address words - Part1: Data format for positioning, line motion and contouring control systems., [s.d.].

ISO/AWI14649-17:2016. Automation systems and integration - Physical device control - Data model for computerized 
numerical controllers - Part 17: Process data for additive manufacturing processes. Retrieved from https://www.din.de/en/getting-involved/standards-committees/nwm/projects/wdc-proj:din21:259391588, [s.d.].

ISO/TS10303-27:2000. Industrial automation systems and integration -- Product data representation and exchange -Part 27: Implementation methods: Java TM programming language binding to the standard data access interface with Internet/Intranet extensions. 2000.

ISO10303-11:2004. Industrial automation systems and integration -- Product data representation and exchange -- Part 11: Description methods: The EXPRESS language reference manual. 2004.

ISO10303-203:2011. Industrial automation systems and integration -- Product data representation and exchange -- Part 203: Application protocol: Configuration controlled 3D design of mechanical parts and assemblies. 2011.

ISO10303-21:2016. Industrial automation systems and integration -- Product data representation and exchange -- Part 21: Implementation methods: Clear text encoding of the exchange structure. 2016.

ISO10303-214:2010. Industrial automation systems and integration -- Product data representation and exchange -- Part 214: Application protocol: Core data for automotive mechanical design processes. 2010.

ISO10303-22:1998. Industrial automation systems and integration -- Product data representation and exchange -- Part 22: Implementation methods: Standard data access interface. 1998.

ISO10303-224:2006. Industrial automation systems and integration -- Product data representation and exchange -- Part 224: Application protocol: Mechanical product definition for process planning using machining features. 2006.

ISO10303-238. Industrial automation systems and integration -- Product data representation and exchange -- Part 238: Application protocol: Application interpreted model for computerized numerical controllers. 2007.

ISO10303-242:2014. Industrial automation systems and integration -- Product data representation and exchange -- Part 242: Application protocol: Managed model-based 3D engineering. 2014.

ISO10303-42:2014. Industrial automation systems and integration -- Product data representation and exchange -- Part 42: Integrated generic resource: Geometric and topological representation. 2014.

JEE, H. S.; LEE, B. Y. Slicing STEP-base CAD models for CAD/RP interface. The SFF Symposium, University of Texas, 1999.

KAI, C. C.; JACOB, G. G. K.; MEI, T. Interface between CAD and Rapid Prototyping systems. Part 2: LMI - An improved interface. The International Journal of Advanced Manufacturing Technology, v. 13, n. 8, p. 571-576, ago. 1997.

KRAMER, T. R. et al. Run-time interpretation of STEP-NC: implementation and performance. International Journal of Computer Integrated Manufacturing, v. 19, n. 6, p. 495-507, set. 2006.

KUMAR, V.; DUTTA, D. An assessment of data formats for layered manufacturing. Advances in Engineering Software, v. 28, n. 3, p. 151-164, 1997.

LASI, H. et al. Industry 4.0. Business \& Information Systems Engineering, v. 6, n. 4, p. 239-242, 19 ago. 2014.

LU, Y.; CHOI, S.; WITHERELL, P. Towards an Integrated Data Schema Design for Additive Manufacturing. Asme, n. August, p. 1-11, 2015.

NASSAR, A. R.; REUTZEL, E. W. A proposed digital thread for additive manufacturing. Solid Freeform Fabrication, p. 19-43, 2013.

NASSEHI, A. NEWMAN, S.T. ALLEN, R. D. STEP-NC compliant process planning as an enabler for adaptive global manufacturing for adaptive global manufacturing. Robotics and Computer-Integrated Manufacturing, n. October, 2006.

NEGI, S.; DHIMAN, S.; SHARMA, R. K. Basics, Applications and Future of Additive Manufacturing Technologies: a Review. Journal of Manufacturing Technology Research, v. 5, n. 1-2, p. 75-96, 2016.

PAVIOT, T. pythonOCC, 3D CAD/CAE/PLM development framework for the Python programming language. Retrieved from http://www.pythonocc.org/quick-examples/step-ap203-import/, 2014.

PRATT, M. The STEP standard as geometry transfer medium for SFF. NSF Workshop on Design Methodologies for Solid Freeform Fabrication, 1995.

PRATT, M. J. Introduction to ISO 10303 - the STEP Standard for Product Data Exchange. Journal of Computing and Information Science in Engineering, v. 1, n. 1, p. 102, 2001. 
RAUCH, M. et al. An advanced STEP-NC controller for intelligent machining processes. Robotics and ComputerIntegrated Manufacturing, v. 28, n. 3, p. 375-384, 2012.

RIAÑO, C. I. et al. Closed-loop Integration Model for Dimensional and Geometric Inspection of Prismatic Parts Based on the STEP-NC standard24th ABCM International Congress of Mechanical Engineering. Anais...Curitiba: 2017

ROCK, S. J.; WOZNY, M. J. A Flexible File Format for Solid Freeform Fabrication. Proceedings Solid Freeform Fabrication Symposium, p. 1-12, 1991.

RODRIGUEZ, E.; RIANO JAIMES, C. I.; ALVARES, A. Projeto Mecatrônico de un Robô com Cinemática Paralela Delta Linear para Manufactura AditivaAnais do IX Congresso Brasileiro de Engenharia de Fabricação. Anais...ABCM, 2017Disponível em: <http://abcm.org.br/anais-de-eventos/COF2017/0282>

RYOU, M. S. et al. Development of a data interface for rapid prototyping in STEP-NC. International Journal of Computer Integrated Manufacturing, v. 19, n. 6, p. 614-626, set. 2006.

SUH, S. H. et al. Architecture and implementation of a shop-floor programming system for STEP-compliant CNC. Computer-Aided Design, v. 35, n. 12, p. 1069-1083, out. 2003.

SZILVŚI-NAGY, M.; MÁTYÁSI, G. Analysis of STL files. Mathematical and Computer Modelling, v. 38, n. 7-9, p. 945-960, out. 2003.

TOQUICA, J. S. et al. A STEP-NC Compliant Robotic Machining Platform for Advanced Manufacturing. International Journal of Advanced Manufacturing Technology, 2017.

XÚ, S.; ANWER, N.; LAVERNHE, S. Conversion of G-code programs for milling into STEP-NC. In: JCM:International Joint Conference on Mechanics, Design Engineering and Advanced Manufacturing, Toulouse, France (2014), p. 495, 2014.

XU, X. W. et al. STEP-compliant NC research: The search for intelligent CAD/CAPP/CAM/CNC integration. International Journal of Production Research, v. 43, n. 17, p. 3703-3743, 2005.

ZENG, K. et al. Layer by Layer Validation of Geometrical Accuracy in Additive Manufacturing processes. Proceedings of Twenty Forth Annual International Solid Freeform Fabrication Symposium - An Additive Manufacturing Conference, n. 1, p. 76-87, 2013.

ZHANG, X. et al. A STEP-compliant process planning system for CNC turning operations. Robotics and ComputerIntegrated Manufacturing, v. 27, n. 2, p. 349-356, abr. 2011. 